\title{
Buckwheat husk processing products as Factor of a Stable Food Market Development
}

\author{
Olga Chugunova, Marina Shkolnikova, and Elena Kadritskaya \\ Ural State University of Economics, 620144, 8 Marta Str., 62, Ekaterinburg, Russia
}

\begin{abstract}
The current state of the economy requires a qualitatively new organization of the region's food market. The development of regional food markets should be based primarily on a fuller use of local raw materials. The most important task in the development of the regional food market is the search for new sources of natural biologically active substances and the development of functional food products based on them. Melanin is a unique biologically active substance with inhibitory, protective, antioxidant, antibacterial, antiviral and antifungal effects. The main plant sources of melanin are chaga, birch mushroom and sunflower husk, the use of which is not always advisable and economically profitable. It is advisable to consider buckwheat husk Fagopyrumesculentum, which is characterized by the absence of ballast fraction and a high content of brown pigment localized in the outer layers of the cellular wall of the entire grain shell, as a promising raw material source for the isolation of melanin. The biochemical characteristics of melanin from alternative raw sources require a more detailed study of the properties of the pigment, in particular the functional ones, to confirm the practical possibility of using them as functional food ingredients in food products - antioxidants and biosorbents-detoxifying agents.
\end{abstract}

\section{Introduction}

The state policy of the Russian Federation is mostly aimed at national security and food security. The social function of the latter is to improve the quality of life, providing high standards of sustenance, i.e. high-quality and safe foodstuff.

Many factors lower body resistance to hazards which disturbs endoecology of a person. Such factors are pollution, man-caused load in some regions of Russia, usage of antibiotics for agricultural, veterinary, and food manufacturing needs. They mostly affect people with weakened immune system, elderly persons, and children. The importance of natural biologically active substances and functional food ingredients with protective, functional and preventive effects is rising for all categories of population, especially children [1].

A step of development for the regional market of raw food and foodstuff is a search of new sources of natural biologically active substances and functional food made of them. Natural ingredients are most advantageous ones as they have a noticeable protective effect and no side effects. Melanin conforms to such demands [1]. All melanin pigments are longchain polymers of high molecular weight and difficult crystal structure [2]. They are highly bioactive. Melanin is proved to have many effects. There is an inhibitory effect on lipid 
peroxygenation by prooxidants [3, 4] and an effect protecting cellular system from mutagenic and cancer-causing factors and disactivating free radicals [5]. Antiviral, antibacterial, antifungal [6], and antioxidant [7] effects are present too. Mechanism of the shielding effect of melanin is due to the ability to absorb free radicals and make inactive complexes [8] from prooxidant ions of transition metals.

Melanin is extracted by chemical and microbiological synthesis or from animal or plant matter. There are two main vegetal sources of melanin - chaga mushroom and sunflower husks. The former is long to reproduce in nature [6]. Usage of the latter may be economically unreasonable because of ballast fractions and a low pigment concentration [7].

Thus, the husks of Fagopyrumesculentum - grain by-products - are a promising raw material resource for melanin extraction. Buckwheat husks have no ballast fraction and are reach in brown pigment concentrated in the outer layers of the shell cell walls.

While melanin is already well-characterized, biochemical features of its pigment extracted from alternative raw materials should be studied thoroughly. So, the purpose of the study is to specify and analyze functional properties of melanin from buckwheat Fagopyrumesculentum husks.

\section{Objects and methods of the study}

The object of the study is water-soluble melanin extracted from husks of buckwheat Fagopyrumesculentum. The husks were cleared, dusted, grinded, and riddled with pores from 1,5 to $4 \mathrm{~mm}$ in diameter. The extraction was conducted in $4 \% \mathrm{NaOH}$ under the water requirement 1:20. One hundred grams of clean and grinded husks were refluxing in the solution of $\mathrm{NaOH}$ and water in a boiling water bath for 2 hours with constant stirring. Then the extract was cooled, the precipitate was filtrated in vacuum. The secondary extraction with conducted likewise under the water requirement 1:10. Filtrates were combined and acidified to $\mathrm{pH}=2$ by $10 \% \mathrm{HCl}$ solution with melanin precipitating. The dark-brown precipitation was filtrated in vacuum by membrane filters "Vladipore membrane MФACOC-2 (Cellulose Acetate membrane micro-filters)", washed with distilled water to the neutral $\mathrm{pH}$ value, and dried under $35^{\circ} \mathrm{C}$ (fig.1).

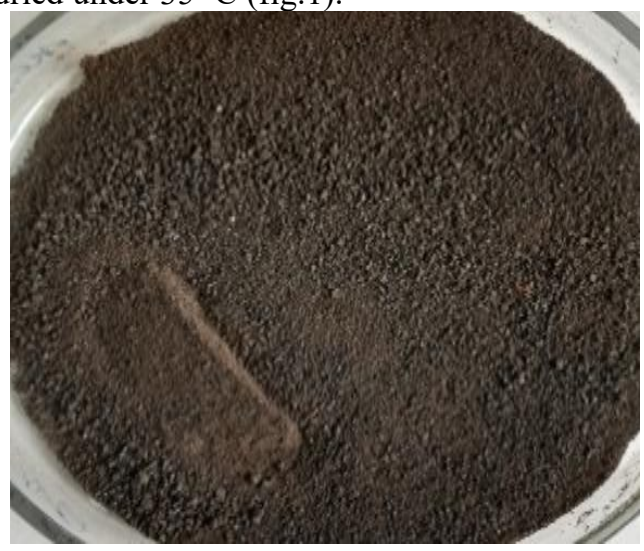

Fig. 1. Melanin extracted from buckwheat husks

Sorption capacity of the melanin extract was found in interaction with copper salt solutions in alkaline condition. The sorption capacity was proved to be maximal in an alkaline condition [9] and not in an acid one. Copper is known to be excreted through the intestinal canal with alkaline condition in course of the metabolism. 
Change of absorbency of the solution was detected in Shimadzu UV-1800 Spectrophotometer. Then a calibration graph was made for the dependence of fixed copper ions concentration on absorbency. Binding capacity was measured in interaction of $10 \mathrm{ml}$ of melanin solution with $4 \% \mathrm{CuSO} 4$ solution. Aqueous solutions of melanin from buckwheat husks were incubated for 1 hour in $\mathrm{T}=37^{\circ} \mathrm{C}$ as in human body. The precipitations obtained were separated by filtration. Absorbency of the solutions was measured with the wavelength at $620 \mathrm{~nm}$.

Investigation of antioxidant activity of the melanin extract sample was conducted by amperometric method for the total antioxidant status in the diapason $0,2-4,0 \mathrm{mg} / \mathrm{dm} 3$. The method involves electric current intensity measuring. The current appears after the substance/ mixture of substances investigated oxidizes on the surface of a working electrode of a certain potential. The signal received is further compared to the standard one of quercetin measured under the same conditions as per GOST R 54037-2010.

\section{Results and discussions}

A most important factor of bioactivity of natural substances is their sorption capacity, i.e. milligrams of ions of a metal bound by one gram of a substance. The capacity of biologically active substances is the reason for them to be used in healthful and dietary meals to cleanse the body of heavy metals.

Lead sorption capacity characteristics are standard for heavy metal analysis of pharmaceutical substances. Copper is also a heavy metal that may contaminate food. The bind of copper and melanin after complexing reaction may be used for preventive care actions against after-effects of excessive ingress of the metal into the body. Copper is known to bind with carboxyl and phosphate groups of biomolecules to lessen enzyme strength and stop metabolic processes.

The results of investigation of melanin sorption capacity to ions of copper are presented in the fig. 2.

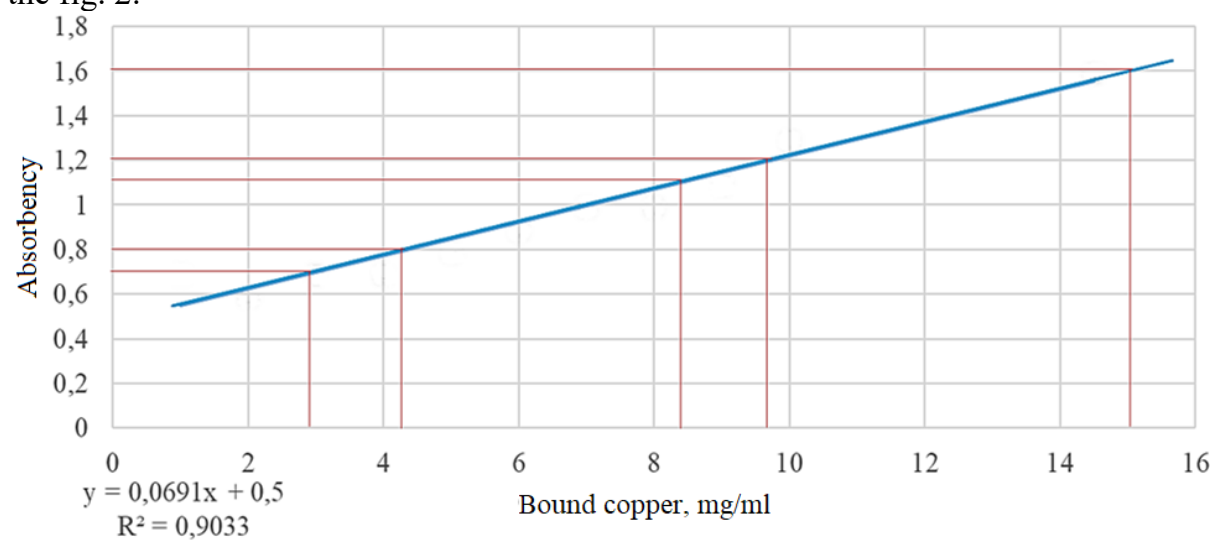

Fig. 2. Amount of copper bound by $0,5 \%$ solution of melanin extract

The data show that the absorbency of the solutions grows as the amount of the melanin extract grows too. Thus, the number of ions of copper in the solution decreases while the amount of the bound copper increases. The proportionality is seen.

The data from the fig. 2 helped to find the amount of copper bound by $0,5 \%$ solution of melanin extract (table 1). 
Table 1. Absorbency of the solutions

\begin{tabular}{|c|c|c|c|c|c|}
\hline Experiment & $\begin{array}{c}\text { 4\% solution } \\
\text { CuSO4, } \mathbf{~ c m}^{\mathbf{3}}\end{array}$ & $\begin{array}{c}\mathbf{0 , 5 \%} \text { solution of } \\
\text { melanin extract, } \\
\mathbf{c m}^{\mathbf{3}}\end{array}$ & $\begin{array}{c}\text { Water, } \\
\mathbf{c m}^{\mathbf{3}}\end{array}$ & Absorbency & $\begin{array}{c}\text { Amount of } \\
\text { bound } \\
\text { copper, } \mathbf{~ m g}\end{array}$ \\
\hline 1 & 2 & 0,0 & 8,0 & 0,7 & 3,5 \\
\hline 2 & 2 & 1,0 & 7,0 & 0,9 & 6,0 \\
\hline 3 & 2 & 2,0 & 6,0 & 1,1 & 8,3 \\
\hline 4 & 2 & 4,0 & 4,0 & 1,2 & 9,5 \\
\hline 5 & 2 & 6,0 & 2,0 & 1,6 & 15,0 \\
\hline
\end{tabular}

So, the higher the absorbency is, the more effective detoxicant the melanin becomes. One gram of $0,5 \%$ solution of the melanin extracted from buckwheat husks bound 966,6 $\mathrm{mg} / \mathrm{ml}$ of copper. Melanin complexes copper due to functional groups. They are hydroxyl (alcohol), phenolic, aminogen, amido, carbonyl groups, fused benzene rings, and phenolic structures [10].

Enough copper in the body improves blood fibrinolytic potential and decreases the cardiovascular risk. The excessive copper builds up in the body leading to many diseases. They are Wilson's disease, liver cirrhosis, or hepatolenticular degeneration. Copper becomes poorly soluble with amino acids and stores in liver or spleen cells, lenticular nucleus, and eye retina. It causes degenerative changes in organs and photophobia. The copper value is 100 times bigger for those with Wilson's disease [11]. Copper value increase in blood is a sign of leukaemia, lymphadenoma, pseudorheumatism, cirrhosis, nephritis.

Chelate of $\mathrm{Fe}$ (II) and $\alpha, \alpha^{\prime}$ dipyridile produced in the process of investigation of melanin extract antioxidant activity was characterized according to its absorbency at 522 $\mathrm{nm}$. The antioxidant activity of $1 \mathrm{~g}$ of melanin extract is equal to the antioxidant activity of $0,056 \mathrm{~g}$ of rutin. The data correspond to the literature ones, i.e. the antioxidant activity of the melanin extract for ascorbic and gallus acids are 0,034 and $0,128 \mathrm{~g}$ respectively [12]. The antioxidant activity of the melanin extract may be due to the reaction with reactive oxygen intermediate or the capacity to use free radicals. Melanin may serve as a natural antioxidant or a food colorant with antioxidant capacities.

\section{Conclusions}

The experiment proves the capacity of melanin extracted from buckwheat husks to bound ions of heavy metals, i.e. copper, in an alkaline condition like that of intestinal canal of human body at $\mathrm{T}=37^{\circ} \mathrm{C}$. Thus, the sample of melanin may be recommended as a detoxicant for salt of heavy metal intoxication and preventative medication for some diseases. The results showed that $1 \mathrm{~g}$ of $0,5 \%$ solution of the melanin extracted from buckwheat husks bound $966,6 \mathrm{mg} / \mathrm{ml}$ of copper and the antioxidant activity of $1 \mathrm{~g}$ of melanin extract is equal to the antioxidant activity of $0,056 \mathrm{~g}$ of rutin. So, the water-soluble samples of melanin extracted from buckwheat husks may be used as antioxidants and biosorbents yet the percentage of melanin is low $(0,05 \%)$. The samples may be also used as food ingredients.

Therefore, the studies are devoted to the idea of a larger use of local raw material resources and development of regional food markets. An important task for regional food market development is to search for new natural biologically active substances and produce functional food of them. So, the results obtained correspond to a relevant social function of the government to improve the quality of life, providing high standards of sustenance, i.e. high-quality and safe foodstuff. 


\section{Ethical practices}

The article carries neither animal nor human subject studies by any of the authors.

\section{References}

1. L. Ya. Rodionova, I.V. Tsiku, New technologies, 3 (2011).

2. F. Solano, Melanins: Skin Pigments and Much More-Types, Structural Models, Biological Functions, and Formation Routes (2014)

3. A. Dontsov, The Protective Effect of Melanins in Oxidative Stress (2014)

4. C. Corsaro, M. Scalia, A. R. Blanco, I. Aiello, G. Sichel, Pigm. Cell Res., 8 (1995)

5. E. A. Prutenskaya, A. S. Vasiliev, E. Yu. Lebedev, I. V. Ushchapovsky, V. A. Silchenko, Symbol of Science, 11 (2016)

6. G. G. Ananko, T. V. Teplyakova, A. V. Bardasheva, T. N. Ilyicheva, Advances in Medical Mycology, 14 (2015)

7. N. V. Gracheva, V. F. Zheltobryukhov, Bulletin of Kazan Technological University, 19 (2016)

8. N. Ushakova, A. Dontsov, N. Sakina, A. Bastrakov, M. Ostrovsky, Biomolecules, 9(9) (2019)

9. L. A. Mikheeva, M. A. Fevraleva, G. T. Brynskikh, A. V. Try, Ulyanovsk Medical Biological J., 2 (2017)

10. E. A. Prutenskaya, A. S. Vasiliev, E. Yu. Lebedeva, I. V. Ushchapovsky, V. A. Silchenko, Symbol of Science, 11-3 (2016)

11. T. Yu. Khaimenova, E. V. Vinnitskaya, I. G. Bakulin, T. V. Shkurko, A. V. Nikitina, Pharmateka, 55 (2016)

12. A. E. Rassabina, O. P. Gurjanov, R. P. Beckett, F. V. Minibayeva, Biochemistry, 85(5) (2020) 